\title{
Clinical validation of a novel quantitative assay for the detection of MGMT methylation in glioblastoma patients
}

\author{
Rocio Rosas-Alonso, PharmD ${ }^{1,2^{*}}$, Julian Colmenarejo-Fernandez, BS ${ }^{1,2}$, Olga Pernia, BS 1, 2 , Carlos \\ Rodriguez-Antolín BS, MS 1, 2, Isabel Esteban, MD, PhD 2,3, Ismael Ghanem, MD, PhD ${ }^{4}$, Dario \\ Sanchez-Cabrero, MD ${ }^{2}$, Itsaso Losantos-Garcia, BS ${ }^{5}$, Javier de Castro, MD, PhD ${ }^{2,4}$, Virginia \\ Martinez-Marin, MD, PhD ${ }^{4}$ e Inmaculada Ibanez-de-Caceres. PhD ${ }^{1,2^{*}}$ \\ 1 Epigenetics Laboratory, INGEMM. La Paz University Hospital, Madrid, Spain \\ 2 Experimental Therapies and Novel Biomarkers in Cancer. IdiPAZ, Madrid, Spain \\ 3 Pathology Department. La Paz University Hospital, Madrid, Spain \\ 4 Medical Oncology Department. La Paz University Hospital, Madrid, Spain \\ 5 Biostatistics Unit. La Paz University Hospital. IdiPAZ.Madrid, Spain
}

* Correspondence: rocio.rosas@salud.madrid.org; inma.ibanezca@salud.madrid.org; Tel:+34-91-2071010-248

\begin{abstract}
The promoter hypermethylation of the methylguanine-DNA methyltransferase (MGMT) gene is a frequently used biomarker in daily clinical practice as it is associated with a favorable prognosis in glioblastoma patients treated with temozolamide. In this study we carried out a clinical validation of a quantitative assay for MGMT methylation detection by comparing a novel MSP custom assay using double-probe characteristics (dp_qMSP) with the conventional MSP in 100 FFPE glioblastoma samples collected from a prospective study in La Paz University Hospital. We realized both determinations and established the best cutoff for the identification of positive-methylated samples using the quantitative data obtained from dp_qMSP. Kaplan-Meier curves and ROC time dependent or ROC(t) curves were employed for the comparison of both methodologies. Our results indicate that the optimal cutoff to categorize the MGMTm positive samples by using dp_qMSP is $3.75 \%$ methylation value. We obtained similar results using both assays in the same cohort of patients, in terms of progression free survival (PFS) and overall survival (OS) when analyzing the Kaplan-Meier curves. The results of ROC(t) curves showed that $d p \_q M S P$ increases the AUC $(t)$ in comparison with MSP for predicting PFS and OS over time. We conclude that dp_qMSP is an alternative methodology compatible with the results obtained with the conventional MSP. This easy-to-use, objective and reliable methodology provides quantitative results and improves the diagnostic precision of patients with glioblastoma in terms of PFS and OS, making it a more competitive assay, suitable for clinical practice.
\end{abstract}

Keywords: MGMT methylation, MSP, dp_qMSP, glioblastoma

\section{Introduction}

Epigenetic modifications are a hallmark of human cancers. The reduction of tumor-associated methylation levels which is associated with genomic instability was one of the first epigenetic alterations to be described [1]. However, there are some areas of the genome that increase their methylation levels, which normally correspond with CpG islands of tumor suppressor genes [2-4]. DNA methylation is catalyzed by DNA methyl-transferases, which transfer methyl groups from S-adenosylmethionine on CpG dinucleotides at the 5'carbon position of cytosines located at CpG islands. Methyl groups are recognized by Methyl-CpG-binding domain proteins, which interfere with the binding of transcriptional activators of DNA [5]. 
The methylguanine-DNA methyltransferase (MGMT) gene promoter hypermethylation is one of the most studied molecular biomarkers in neuro-oncology. MGMT gene encodes a repair enzyme that removes alkyl groups from the 06 position of guanine and works by antagonizing the cytotoxic effects of alkylating agents [6]. Promoter methylation is the main way of silencing the MGMT gene and predicts a favorable outcome in glioblastoma patients treated with alkylating drugs. Glioblastoma (GBM) is the most common primary malignant central nervous system tumor in adults and is invariably associated with poor prognosis. Only 33\% of patients survive the one year mark and only $5 \%$ of patients live more than five years after diagnosis [7-9]. Thus, the methylation status of MGMT is frequently used in the daily clinical routine as a predictive biomarker to classify GBM patients who are more likely to respond to temozolamide.

The MGMT CpG island has 98 CpG sites located on chromosome 10q26 that controls the MGMT gene expression. Malley et al. defined a differentially methylated region (DMR2) essential for silencing the MGMT gene. Most of the assays are based on the analysis of the CpG sites 73 to 90 located at the DMR2 area. Throughout this area, the CpGs 83, 86, 87 and 89 have been the best targets for methylation testing [10]. Furthermore, Bady et al. described two CpG sites in the MGMT promoter (cg12434587, chr10:131265209-131265210 and cg12981137, chr10:131265575-131265576) that showed the strongest association with overall survival (OS), being cg12981137 the CpG number 84 in the DMR2 area, and supporting the idea proposed by Malley et al. [11].

A wide range of molecular assays are available for qualitative and quantitative MGMT methylation detection. The most commonly used methods are based on bisulfite conversion of unmethylated cytosines into uracil [12]. Examples of methods include methylation-specific PCR (MSP) [6,13,14], pyrosequencing [13-15], different variations of real-time PCR [14,16], digital PCR [17], methylation-specific multiplex ligation-dependent probe amplification (MS-MLPA) [13,18], methylation-specific high-resolution melting (HRM) [19], and combined bisulfite restriction analysis (COBRA) [20]. Other techniques that can evaluate global methylation changes such as next-generation sequencing are currently employed in the field of research but not in the routine clinical practice [21].Currently, MSP and pyrosequencing are the most widely used technical approaches to MGMT methylation analysis, providing information that is useful for clinical decision-making. However, the analytical sensitivity differs considerably among diverse assays and their standardization across a wide range of diagnostic laboratories is lacking [22]. In fact, there is still a lack of consensus on how to interpret the pyrosequencing data $[14,15]$. In addition to the method used, other factors such as tumor content, contamination of inflammatory and stromal cells, necrosis, and tumor heterogeneity could affect the methylation results obtained [23].

Due to the increasing interest in molecular biomarkers and their impact in therapeutic management of glioblastoma patients, more sensitive and competitive alternative methodologies are in demand. In this study, we have developed an innovative quantitative methylation specific PCR (dp_qMSP) assay used for the study of MGMT methylation and validated its clinical use by comparing this novel assay with the conventional MSP.

\section{Results}

This section may be divided by subheadings. It should provide a concise and precise description of the experimental results, their interpretation as well as the experimental conclusions that can be drawn.

\subsection{Clinical data}

From May 2014 to March 2020, we enrolled 100 patients with newly diagnosed GBM. Among the 100 patients, 42 were women and 58 were men. The average age at diagnosis was 61 years old (age range: $24-83$ years).

No significant differences were found between patients' age, sex, type of surgery, ECOG and MGMT promoter methylation assessed with MSP or dp_qMSP. Relevant clinical data of patients are described in Table 1. 
Table 1. Demographic and clinical data of the study population $(n=100)$.

\begin{tabular}{|c|c|c|c|}
\hline Characteristic & Value & MSP (p-value) & dp_qMSP (p-value) \\
\hline $\begin{array}{c}\text { Average age at surgery } \\
\text { and range }\end{array}$ & $\begin{array}{c}61 \\
(25-84)\end{array}$ & $p=0.632$ & $p=0.697$ \\
\hline \multicolumn{4}{|l|}{ Sex } \\
\hline Women & 42 & \multirow{2}{*}{$p=0.377$} & \multirow{2}{*}{$p=0.218$} \\
\hline Men & 58 & & \\
\hline \multicolumn{4}{|l|}{ Type of surgery } \\
\hline Total resection & 51 & \multirow{3}{*}{$p=0.174$} & \multirow{3}{*}{$p=0.367$} \\
\hline Partial resection & 29 & & \\
\hline Biopsy & 20 & & \\
\hline \multicolumn{4}{|l|}{ ECOG } \\
\hline 0 & 59 & \multirow{4}{*}{$p=0.727$} & \multirow{4}{*}{$p=0.624$} \\
\hline 1 & 24 & & \\
\hline 2 & 12 & & \\
\hline 3 & 5 & & \\
\hline
\end{tabular}

\subsection{Comparison between dp_qMSP and MSP methods for MGMT promoter methylation detection}

ROC curve was performed to determine the cutoff for dp_qMSP. The area under the curve (AUC) was 0.962 (95\% CI: 0.927-0.998) (Figure 1). When the cutoff methylation point was 3.75\%, the sensitivity and specificity were 100\% (95\% CI: 88.6-100) and 88.6\% (95\% CI: 79.0-94.1), respectively. Thus, the samples were classified as methylated when the methylation was above the cutoff point of $3.75 \%$ and unmethylated when they were less than 3.75\%. MGMT methylation was detected in 30 out of 100 FFPE samples by MSP and 38 out of 100 samples by dp_qMSP (Table 2); a representative gel and quantitative amplifications are shown in Figure 2 (see the uncropped gel at Supplementary Figure 1). If we consider the eight positives identified by dp_qMSP to be false positives based on the data obtained using MSP, the specificity achieved by dp_qMSP would be $88.6 \%$.

Table 2. Clinical, pathological and methylation data of 100 GBM patients

\begin{tabular}{ccccccc}
\hline ID & Age & Sex & Type of surgery & ECOG & MSP & dp_qMSP \\
\hline $\mathbf{1}$ & 57 & Female & Total resection & 0 & $\mathrm{U}$ & 66,6 \\
$\mathbf{2}$ & 70 & Male & Biopsy & 2 & $\mathrm{U}$ & 0 \\
$\mathbf{3}$ & 45 & Male & Partial resection & 0 & $\mathrm{M}$ & 65 \\
$\mathbf{4}$ & 44 & Male & Partial resection & 0 & $\mathrm{U}$ & 0 \\
$\mathbf{5}$ & 54 & Male & Partial resection & 0 & $\mathrm{U}$ & 0 \\
$\mathbf{6}$ & 71 & Male & Total resection & 0 & $\mathrm{U}$ & 1,6 \\
$\mathbf{7}$ & 61 & Female & Total resection & 0 & $\mathrm{M}$ & 60,3 \\
$\mathbf{8}$ & 47 & Female & Total resection & 0 & $\mathrm{M}$ & 66 \\
$\mathbf{9}$ & 77 & Female & Biopsy & 3 & $\mathrm{U}$ & 0 \\
$\mathbf{1 0}$ & 49 & Male & Biopsy & 0 & $\mathrm{U}$ & 0 \\
$\mathbf{1 1}$ & 69 & Female & Partial resection & 2 & $\mathrm{M}$ & 5,9 \\
$\mathbf{1 2}$ & 67 & Male & Total resection & 0 & $\mathrm{U}$ & 0 \\
$\mathbf{1 3}$ & 65 & Female & Total resection & 0 & $\mathrm{U}$ & 0 \\
$\mathbf{1 4}$ & 80 & Female & Total resection & 0 & $\mathrm{U}$ & 0 \\
\hline
\end{tabular}




\begin{tabular}{|c|c|c|c|c|c|c|}
\hline 15 & 66 & Female & Partial resection & 0 & $\mathrm{U}$ & 0 \\
\hline 16 & 65 & Female & Biopsy & 1 & $\mathrm{U}$ & 0 \\
\hline 17 & 81 & Female & Biopsy & 0 & $\mathrm{U}$ & 0 \\
\hline 18 & 72 & Male & Partial resection & 0 & M & 97,5 \\
\hline 19 & 54 & Male & Partial resection & 3 & $\mathrm{U}$ & 0 \\
\hline 20 & 51 & Male & Total resection & 0 & $\mathrm{U}$ & 0 \\
\hline 21 & 49 & Male & Total resection & 3 & $\mathrm{U}$ & 0 \\
\hline 22 & 79 & Female & Total resection & 2 & M & 99,9 \\
\hline 23 & 55 & Female & Partial resection & 1 & $\mathrm{U}$ & 0 \\
\hline 24 & 64 & Male & Total resection & 0 & M & 27,5 \\
\hline 25 & 49 & Male & Total resection & 0 & M & 94,1 \\
\hline 26 & 62 & Female & Total resection & 0 & M & 97,6 \\
\hline 27 & 55 & Male & Partial resection & 0 & $\mathrm{U}$ & 0 \\
\hline 28 & 65 & Male & Partial resection & 0 & $\mathrm{U}$ & 64,6 \\
\hline 29 & 58 & Male & Partial resection & 0 & $\mathrm{U}$ & 0 \\
\hline 30 & 71 & Female & Total resection & 0 & $\mathrm{U}$ & 0 \\
\hline 31 & 76 & Female & Total resection & 0 & M & 17,8 \\
\hline 32 & 73 & Female & Total resection & 0 & M & 83 \\
\hline 33 & 67 & Male & Total resection & 0 & $\mathrm{U}$ & 0 \\
\hline 34 & 60 & Male & Total resection & 0 & $\mathrm{U}$ & 0 \\
\hline 35 & 50 & Female & Total resection & 0 & M & 36 \\
\hline 36 & 54 & Male & Total resection & 0 & M & 96,4 \\
\hline 37 & 62 & Male & Biopsy & 0 & $\mathrm{U}$ & 0 \\
\hline 38 & 76 & Female & Biopsy & 0 & M & 100 \\
\hline 39 & 76 & Female & Total resection & 1 & $\mathrm{U}$ & 0 \\
\hline 40 & 84 & Male & Biopsy & 0 & $\mathrm{U}$ & 0 \\
\hline 41 & 75 & Male & Total resection & 1 & M & 100 \\
\hline 42 & 46 & Male & Total resection & 0 & $\mathrm{U}$ & 0 \\
\hline 43 & 55 & Female & Partial resection & 2 & $\mathrm{U}$ & 0 \\
\hline 44 & 80 & Female & Partial resection & 1 & $\mathrm{U}$ & 0 \\
\hline 45 & 29 & Male & Biopsy & 0 & M & 77 \\
\hline 46 & 56 & Male & Biopsy & 1 & $\mathrm{U}$ & 0 \\
\hline 47 & 61 & Male & Total resection & 1 & $\mathrm{U}$ & 0 \\
\hline 48 & 72 & Male & Total resection & 0 & M & 92,6 \\
\hline 49 & 48 & Female & Partial resection & 1 & M & 100 \\
\hline 50 & 66 & Male & Total resection & 0 & $\mathrm{U}$ & 0 \\
\hline 51 & 70 & Male & Partial resection & 0 & $\mathrm{U}$ & 0 \\
\hline 52 & 82 & Male & Biopsy & 3 & M & 99,9 \\
\hline 53 & 52 & Female & Total resection & 0 & $\mathrm{U}$ & 0 \\
\hline 54 & 54 & Female & Total resection & 0 & M & 100 \\
\hline 55 & 68 & Female & Biopsy & 2 & $\mathrm{U}$ & 0 \\
\hline 56 & 60 & Male & Total resection & 0 & $\mathrm{U}$ & 0 \\
\hline 57 & 54 & Male & Partial resection & 0 & $\mathrm{U}$ & 0 \\
\hline
\end{tabular}




\begin{tabular}{|c|c|c|c|c|c|c|}
\hline 58 & 64 & Male & Partial resection & 0 & $\mathrm{U}$ & 0 \\
\hline 59 & 75 & Female & Total resection & 0 & M & 88,89 \\
\hline 60 & 68 & Female & Partial resection & 1 & $\mathrm{U}$ & 0 \\
\hline 61 & 73 & Female & Partial resection & 1 & $\mathrm{U}$ & 0 \\
\hline 62 & 51 & Male & Total resection & 1 & $\mathrm{U}$ & 0 \\
\hline 63 & 37 & Male & Total resection & 1 & M & 100 \\
\hline 64 & 69 & Male & Biopsy & 0 & $\mathrm{U}$ & 0 \\
\hline 65 & 71 & Male & Total resection & 0 & M & 100 \\
\hline 66 & 67 & Female & Total resection & 1 & $\mathrm{U}$ & 0 \\
\hline 67 & 51 & Male & Total resection & 2 & M & 99,9 \\
\hline 68 & 50 & Male & Total resection & 0 & $\mathrm{U}$ & 0 \\
\hline 69 & 57 & Female & Biopsy & 0 & $\mathrm{U}$ & 0 \\
\hline 70 & 50 & Female & Total resection & 0 & $\mathrm{U}$ & 0,4 \\
\hline 71 & 61 & Male & Total resection & 1 & $\mathrm{U}$ & 0 \\
\hline 72 & 56 & Male & Biopsy & 0 & $\mathrm{U}$ & 0 \\
\hline 73 & 73 & Male & Total resection & 2 & $\mathrm{U}$ & 0 \\
\hline 74 & 49 & Male & Total resection & 0 & $\mathrm{U}$ & 0 \\
\hline 75 & 63 & Female & Partial resection & 1 & $\mathrm{U}$ & 13 \\
\hline 76 & 60 & Male & Partial resection & 0 & $\mathrm{U}$ & 81 \\
\hline 77 & 71 & Male & Total resection & 0 & $\mathrm{U}$ & 0 \\
\hline 78 & 65 & Male & Total resection & 2 & $\mathrm{U}$ & 99,9 \\
\hline 79 & 64 & Male & Total resection & 0 & $\mathrm{U}$ & 0 \\
\hline 80 & 40 & Female & Partial resection & 0 & $\mathrm{U}$ & 0 \\
\hline 81 & 79 & Male & Total resection & 1 & $\mathrm{U}$ & 0 \\
\hline 82 & 44 & Female & Total resection & 2 & M & 100 \\
\hline 83 & 51 & Male & Total resection & 1 & M & 48,6 \\
\hline 84 & 62 & Male & Total resection & 0 & $\mathrm{U}$ & 0 \\
\hline 85 & 62 & Male & Total resection & 0 & $\mathrm{U}$ & 0 \\
\hline 86 & 62 & Female & Partial resection & 0 & $\mathrm{U}$ & 0 \\
\hline 87 & 71 & Female & Biopsy & 1 & $\mathrm{U}$ & 0 \\
\hline 88 & 66 & Male & Total resection & 1 & $\mathrm{U}$ & 0 \\
\hline 89 & 57 & Male & Biopsy & 0 & $\mathrm{U}$ & 35 \\
\hline 90 & 69 & Male & Partial resection & 1 & M & 94,4 \\
\hline 91 & 67 & Female & Biopsy & 1 & $\mathrm{U}$ & 6,9 \\
\hline 92 & 59 & Male & Partial resection & 0 & $\mathrm{U}$ & 0 \\
\hline 93 & 57 & Female & Total resection & 2 & $\mathrm{U}$ & 0 \\
\hline 94 & 52 & Male & Partial resection & 0 & $\mathrm{U}$ & 0 \\
\hline 95 & 50 & Male & Partial resection & 1 & M & 87,8 \\
\hline 96 & 60 & Female & Partial resection & 2 & M & 91,3 \\
\hline 97 & 58 & Male & Biopsy & 2 & $\mathrm{U}$ & 0 \\
\hline 98 & 74 & Female & Partial resection & 1 & M & 47,9 \\
\hline 99 & 25 & Female & Biopsy & 3 & $\mathrm{U}$ & 0 \\
\hline 100 & 57 & Female & Total resection & 1 & $\mathrm{U}$ & 100 \\
\hline
\end{tabular}




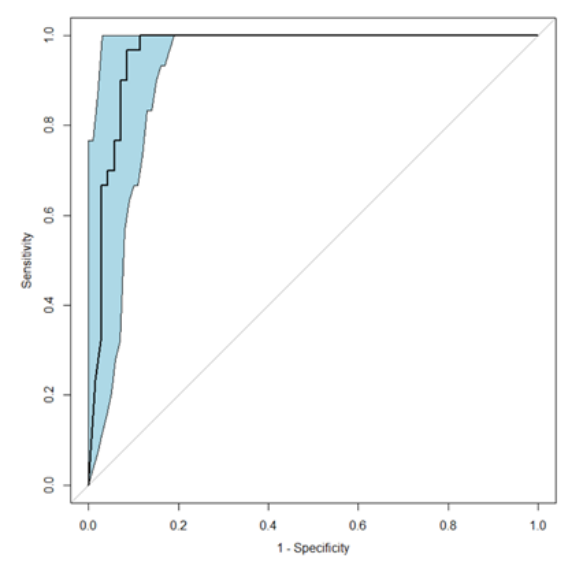

Figure 1. ROC curve for dp_qMSP compared to MSP $(n=100)$. Area under the curve $=0.962(95 \%$ CI: 0.9268-0.998). Blue shade represents the CI.

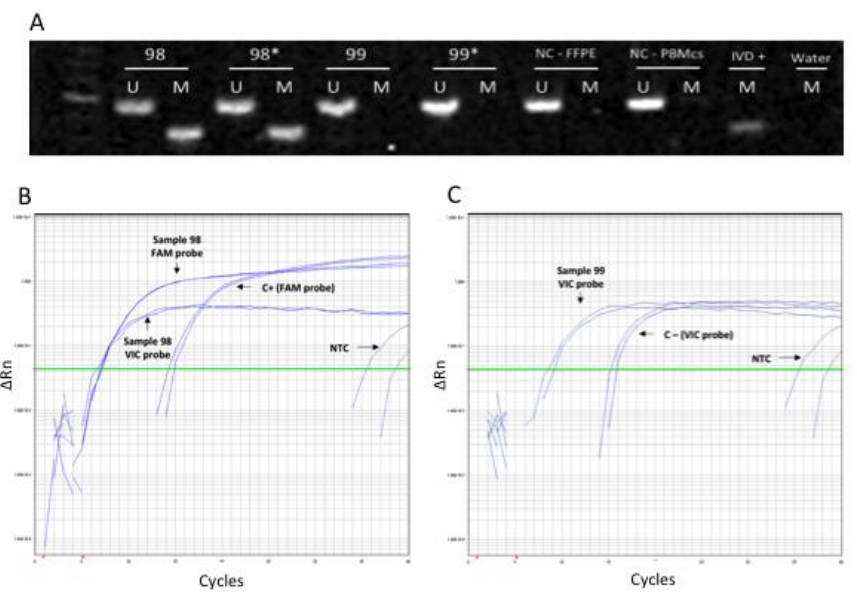

Figure 2. MSP and dp_qMSP examples in the analyzed tumor samples for patients 98 and 99. A. Example of MGMT promoter methylation in acrylamide gel. The MSP products were loaded and electrophoresed as follows: Sample number 98 (lanes 1-4 unmethylated and methylated reactions performed by duplicated), Sample number 99 (lanes 5-8, unmethylated and methylated reactions performed by duplicated), lanes 9-10 correspond to unmethylated and methylated reactions using a FFPE negative control sample. Lanes 11 and 12 correspond to unmethylated and methylated reactions using a PBMC control sample. Lane 13 corresponds to a positive control (IVD) and last line is the water methylation reaction used to discard contamination. B and C. Example of methylated and unmethylated amplification by qMSP. B. Patient number 98, FAM and VIC probes amplified (47,9\% methylation). C. Patient number 99, only VIC probe amplified (0\% methylation). U: Unmethylated. M: methylated. FFPE: Formalin fixed paraffin embedded. PBMCs: Peripheral blood mononuclear cells. NC: negative control. IVD: In vitro Methylated DNA (positive control). NTC (No Template Control).

\subsection{Examination dp_qMSP and MSP methods for survival analysis}


The median of OS measured by MSP in the group of patients with unmethylated MGMT promoter in our cohort was 11.8 months (95\% CI: 10.4 to 13.2) while the median of OS was not reached in the methylated group (Figure 3A). We observed significant differences between unmethylated and methylated groups in terms of OS ( $\mathrm{p}=0.004, \mathrm{HR}=0.37,95 \%$ CI: 0.19 to 0.72 ). The rate of OS at two years was only $17 \%$ in the unmethylated group compared with the $53 \%$ observed in the methylated group. The median PFS was 7.0 months (95\% CI: 5.3 to 8.8) in the unmethylated MSP group and 18.0 months (95\% CI: 9.8 to 26.1) in the methylated MSP group (Figure 3B). We also observed significant differences in terms of PFS regarding the methylation status between groups ( $p<0.001, \mathrm{HR}=0.33,95 \%$ CI: 0.18 to 0.61 ). The rate of PFS at two years was $9.2 \%$ in the unmethylated group compared to the $31.5 \%$ observed in the methylated group. When using MGMT methylation data obtained by qMPS, the median OS in the unmethylated group was 12.6 months (95\% CI: 10.0 to 15.1) while this median was not reached in the methylated group (Figure 3C). Consistent with the results obtained by MSP, there were significant differences between unmethylated and methylated groups in terms of OS ( $\mathrm{p}=0.014, \mathrm{HR}=0.47,95 \% \mathrm{CI}$ : 0.26 to 0.86 ) and PFS ( $\mathrm{p}=0.001, \mathrm{HR}=0.41,95 \% \mathrm{CI}$ : 0.24 to 0.70 ). The rate of OS at two years was $19 \%$ in the unmethylated group compared to $45 \%$ in the methylated group. The median PFS was 7.0 months (95\% CI: 5.6 to 8.4 ) in the unmethylated dp_qMSP group and 16.0 months (95\% CI: 11.8 to 20.3) in the methylated dp_qMSP group (Figure 3D). While the rate of PFS at two years was $10.8 \%$ in the unmethylated group compared to the $24.9 \%$ observed in the methylated group.
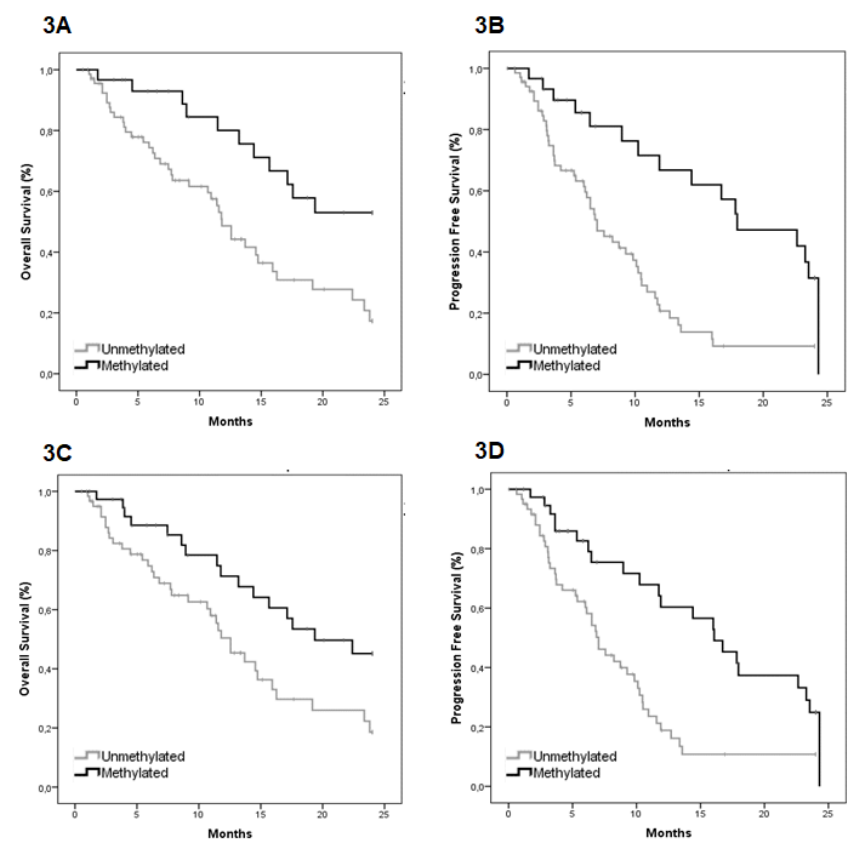

Figure 3. Survival analysis of GBM patients. A: Kaplan-Meier OS graph comparing methylation GBM patients to unmethylated classified according to MSP. B: Kaplan-Meier PFS graph comparing methylation GBM patients to unmethylated classified according to MSP. C: Kaplan-Meier OS graph comparing methylation GBM patients to unmethylated classified according to dp_qMSP. D: Kaplan-Meier PFS graph comparing methylation GBM patients to unmethylated classified according to MSP.

\subsection{Comparison between $d p \_q M S P$ and MSP methods for progression evaluation according to $R O C(t)$}

We performed ROC(t) curves to compare both MSP and dp_qMSP for predicting PFS and OS in our cohort of GBM patients. The time-dependent area under the curve or $\mathrm{AUC}(\mathrm{t})$ for OS was 0.49 when we analyzed the patients with the MSP method and 0.60 in dp_qMSP assay $(p=0.001)$. The 
AUC(t) for PFS was 0.50 when we analyzed the patients with MSP method and 0.58 in dp_qMSP assay ( $\mathrm{p}=0.037$ ) (Figure 4$)$.
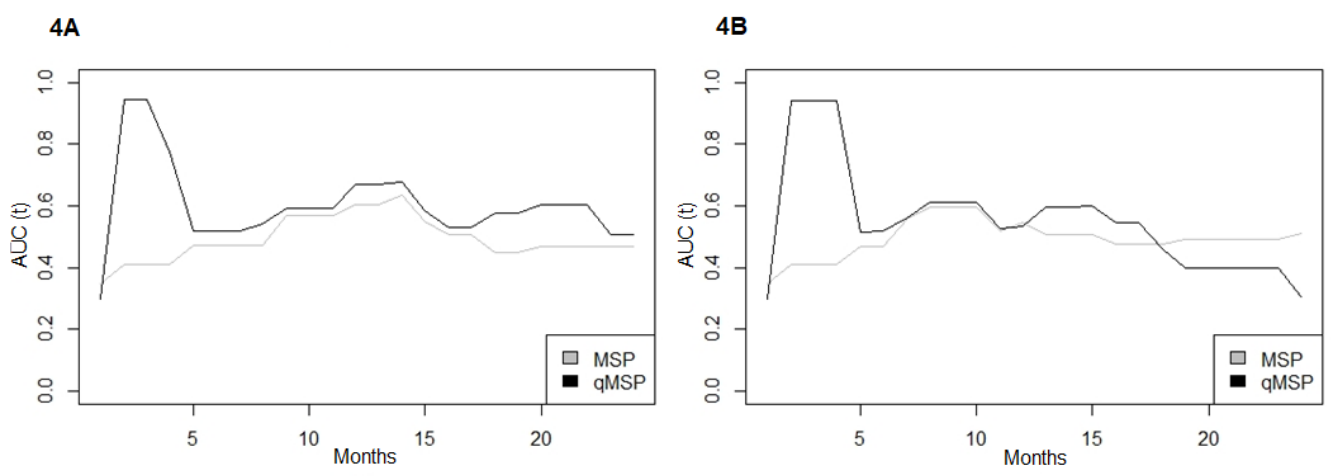

Figure 4. ROC (t) curves predicting OS and PFS. A: AUC(t) for OS is higher for dp_qMSP than $\operatorname{MSP}(p=0.001)$. B: AUC $(t)$ for PFS is higher for dp_qMSP than MSP $(p=0.037)$.

\section{Discussion}

Food and Drug Administration and National Institutes of Health Biomarker Working Group define validation as a process to establish that the performance of a test is acceptable for its intended purpose [29]. In order to establish if the dp_qMSP test is suitable for MGMT methylation analysis, we performed comparison-of-methods studies between both dp_qMSP and MSP processes.

Firstly, we carried out a ROC study establishing the best cutoff methylation point at $3.75 \%$ and finding an excellent model (AUC $=0.962$ ). The sensitivity obtained was $100 \%$ and we detected eight additional positive samples that were not identified by MSP. Being strict, we considered them as false-positive, decreasing our specificity to $88.6 \%$; although they could certainly be due to an increased sensitivity of our methodology compared to the MSP. In fact, qPCR assays combine PCR chemistry with amplicon detection by a fluorescence probe with a MGB, which stabilizes the double-stranded probe template structure resulting in improved allele specificity [30]. Moreover, qPCR can exclude ambiguity of interpretation which may cause bias in conventional PCR and it presents an easier workflow [31]. Thus, if we take into account that qPCR has higher sensitivity than the SYBERgreen-stained and gel-based detection under ultraviolet light we should not consider the new methylated samples identified by dp_qMSP to be false positives when using dp_qMSP, but rather that they are false negatives when using MSP. With this in mind, we carried out further studies in order to decide the adequacy of both assays in terms of OS and PFS.

The Kaplan-Meir analysis showed that patients with MGMT promoter methylation resulted in significantly longer PFS and OS than unmethylated patients, the same results as previously reported independently of using MSP or dp_qMSP [7,14]. Therefore, both techniques allow the classification of patients as responders or non-responders in terms of MGMT methylation.

However, the question to be addressed is how well does the MGMT methylation biomarker evaluated by dp_qMSP distinguish between patients who respond to treatment and patients who do not at a given follow-up time. Cancer outcomes are very time-dependent and ROC curves that vary as a function of time may be more useful that the Kaplan-Meir analysis [28]. Therefore, ROC ( $t$ ) has been used to compare the MSP and dp_qMSP and to establish the one that best fits the survival data. The AUC ( $t$ ) for OS and PFS obtained was higher when we used the dp_qMSP method ( 0.49 versus 0.60 in OS and 0.50 versus 0.58 in PFS). We found significant differences between MSP and dp_qMSP, suggesting that the dp_qMSP assay might be more effective at detecting the MGMT methylation biomarker than the classic MSP assay.

In addition to the aforementioned advantages of using the dp_qMSP method, we may have obtained better results in AUC ( $t$ ) particularly within the first 5 months after diagnosis in dp_qMSP 
because we are investigating the most important positions that have been shown to have a major impact on MGMT expression. Malley et al described the methylation status of CpGs 83, 86, 87 and 89 as critical for transcriptional regulation, being the CpG 83 in our hydrolysis probe and CpG 86 and 87 in reverse primer. In addition, we have in our reverse primer the cg12981137, described by Bady et al as one of the two more essential [10,11] (Figure 5).

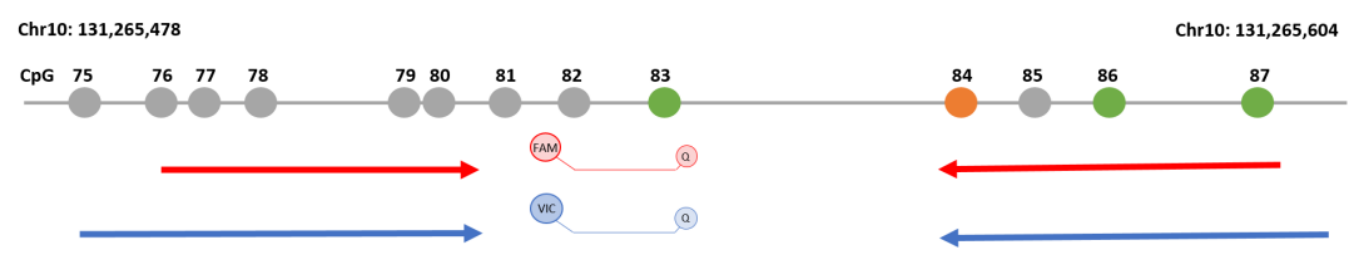

Figure 5. ROC $(t)$ curves predicting OS and PFS. A: AUC( $(t)$ for OS is higher for dp_qMSP than $\operatorname{MSP}(p=0.001)$. B: AUC $(t)$ for PFS is higher for dp_qMSP than MSP $(p=0.037)$.

Due to the absence of adequately standardized techniques, international harmonization of the MGMT biomarker is still an unmet clinical need. A main difficulty has been the lack of a gold standard for MGMT methylation detection. We used MSP as a reference because it was the first method described and has been repeatedly shown to be of predictive value in randomized clinical trials [32-35].

However, several studies reported pyrosequencing as the method of choice for MGMT promoter methylation analysis in routine clinical practice [14,36,37]. The current limitation of pyrosequencing is the absence of a consensus regarding an established cutoff for binary classification and concerning which are the most relevant $\mathrm{CpG}$ sites to analyze for clinical practice (as there are several pyrosequencing protocols that differ in regards to the number and position of the studied CpG sites) [15,38-40]. For these reasons, MGMT methylation status has sometimes suffered from inconsistent results in the same tumor with different methods, mainly due to the lack of methodological standardization.

\section{Materials and Methods}

\subsection{Patient samples}

Formalin-fixed paraffin-embedded (FFPE) GBM samples from 100 patients prospectively collected at University hospital La Paz, who underwent surgery or biopsy at the La Paz University Hospital between May 2014 and March 2020 were included in this study. The percentage of tumor cells in the tissue was evaluated previously by an expert pathologist to ensure the quality of the tumor sample. Inclusion criteria encompassed patients with signed informed consent, 18 years of age or older, Eastern Cooperative Oncology Group (ECOG) performance status of 0 to 3 , histologically confirmed as a IDH-wild type GBM and treated with temozolamide. All patients had a minimum follow-up of 3 months.

The demographic information of all the patients as well as type of surgery, treatment, progression-free survival (PFS) and overall survival (OS) were collected. This study was conducted under the approval of the ethics committee of the La Paz University Hospital with the ethics number of PI-2887 and in conformance with the principles of the Declaration of Helsinki. Written informed consent was obtained from all participants.

\section{.4.2. Nucleic acid isolation}


Formalin-fixed and paraffin-embedded (FFPE) tissue samples were deparaffinized using xylene. DNA from tissue samples and cells were extracted according to standard protocols using phenol-chloroform, chloroform and finally suspending in $20 \mu \mathrm{l}$ of $1 \mathrm{X}$ TE [24].

\subsection{Bisulfite modification of DNA}

$1000 \mathrm{ng}$ of DNA from plasma was denatured by $\mathrm{NaOH}(0.2 \mathrm{~mol} / \mathrm{L})$ for 10 minutes at $37^{\circ} \mathrm{C}$ and then modified by hydroquinone and sodium bisulfite treatment at $50^{\circ} \mathrm{C}$ for 17 hours. Modified DNA was purified using the Wizard DNA Clean-Up system (Promega, Madison, WI). Modification was completed by $\mathrm{NaOH}(0.3 \mathrm{~mol} / \mathrm{L})$ treatment for five minutes at room temperature followed by precipitation with glycogen, $10 \mathrm{~mol} / \mathrm{L}$ ammonium acetate, and ethanol precipitation. Bisulfite modification of DNA resulted in the conversion of unmethylated cytosines to uracil, whereas methylated cytosines were resistant to modification and remained as cytosine [25]

\subsection{Nested-PCR}

We performed a nested PCR to improve sensitivity and specificity. The reaction mixture contained $1.1 \mu \mathrm{l}$ of bisulfite-modified DNA, $3 \mu \mathrm{l}$ buffer (Biotools Buffer 10X), $4 \mu \mathrm{l}$ dNTPs (10 mM), $1 \mu \mathrm{l}$ $\mathrm{MgCl} 2(50 \mathrm{nM}), 2 \mu \mathrm{l}$ primers $(100 \mathrm{ng} / \mu \mathrm{L})$ and $0.75 \mu \mathrm{l}$ (Biotools DNA polymersa $1 \mathrm{U} / \mu \mathrm{l})$ enzyme in a total reaction volume of $20.5 \mu$ l. Nested PCR conditions were $94^{\circ} \mathrm{C}$ for $5 \mathrm{~min}$, followed by 40 cycles at $94{ }^{\circ} \mathrm{C}$ for one $\mathrm{min}, 54{ }^{\circ} \mathrm{C}$ for one $\mathrm{min}, 72{ }^{\circ} \mathrm{C}$ for one min and $72^{\circ} \mathrm{C}$ for eight min. The product of the reaction is a $274 \mathrm{bp}$ amplicon. The sequences (5'-3') used were as follows: forward primer (BS_F) GGATATGTTGGGATAGTT and reverse primer (BS_R) CCGAAAAAAAACTAAACAACACCT. Amplicons resulting from this PCR reaction were used as template for MSP and dp_qMSP.

\subsection{Methylation-specific PCR (MSP)}

The MGMT methylation status of patients' samples was determined in the clinical routine by MSP. Consequently, amplicons resulting from nested-PCR were amplified using specific primers that discriminate methylated (M) and unmethylated (U) DNA previously described by Esteller et al [26].

Primer sequences for $\mathrm{M}$ and U PCR reactions are as follows: for the methylated sequence MGMT_MF, TTTCGACGTTCTAGGTTTTCGC; MGMT_MR: GCACTCTTCCGAAAACGAAACG and for the unmethylated sequence MGMT_UF, TTTGTGTTTTGATGTTTGTAGGTTTTTGT; MGMT_UR, AACTCCACACTCTTCCAAAAACAAAACA. Primers for U reactions were analogous to $M$ reactions, except $C G$ was replaced with TG in the forward primer or $C A$ in reverse primers. In addition, the $U$ primers have additional base pairs, to be able to distinguish both reactions in an acrylamide gel. Primers were designed for the detection of an $81 \mathrm{bp}$ fragment obtained from the methylated reaction and a $93 \mathrm{bp}$ fragment from the unmethylated reaction. Peripheral blood mononuclear cell (PBMC) methylated in vitro with the enzyme CpG-Methyltransferase (M.SssI) was used as the methylated control.

The MSP reaction was performed in a final volume of $25 \mu \mathrm{l}$ containing $1.1 \mu \mathrm{l}$ of amplicons resulting from nested-PCR, $4.4 \mu \mathrm{l} \mathrm{dNTPs}(10 \mathrm{mM}), 0.4 \mu \mathrm{l}$ of each primers $(100 \mathrm{ng} / \mu \mathrm{L}), 0.5 \mu \mathrm{l} \mathrm{DNA}$ polymerase (Biotools DNA polymersa $1 \mathrm{U} / \mu \mathrm{l}$ ) and $11.3 \mu \mathrm{l} \mathrm{H} 2 \mathrm{O}$. The MSP reaction was carried out with the following settings: for $\mathrm{M}$ reaction, $37 \mathrm{cycles}$ of $95^{\circ} \mathrm{C}$ for one $\mathrm{min}, 68^{\circ} \mathrm{C}$ for one min, and $72^{\circ} \mathrm{C}$ for 50 $\mathrm{s}$; for $\mathrm{U}$ reaction, 35 cycles of $95^{\circ} \mathrm{C}$ for one min, $60^{\circ} \mathrm{C}$ for one min, and $72^{\circ} \mathrm{C}$ for $50 \mathrm{sec}$. The MSP products were electrophoresed on a $6 \%$ non-denaturing acrylamide gels with appropriate size markers and the presence or absence of a PCR product analyzed under ultraviolet light.

\subsection{Quantitative methylation-specific PCR (dp_qMSP)}

MGMT-dp_qMSP is a customized quantitative real-time PCR (qPCR) that specifically detects methylated and unmethylated bisulfite-modified DNA molecules from the same chromosomic location, specifically at the MGMT promoter area.

The dp_qMSP assay was carried out using ABI Prism 7900HT (Applied Biosystems, Darmstadt, Germany). We have used the same primers as those used for the MSP. In addition, we designed two 
hydrolysis probes, specifically for methylated or unmethylated DNA molecules. The methylated MGMT hydrolysis probe was labelled with a FAM fluorochrome at the $5^{\prime}$ end (FAM-CAAATCGCAAACGATA-MGB-NFQ) and the unmethylated MGMT hydrolysis probe was labelled with a VIC fluorochrome at the 5' end (VIC-CAAATCACAAACAATA-MGB-NFQ). Both probes have a non-fluorescentquencher (NFQ) with a minor groove binder (MGB) at the $3^{\prime}$ end. Hydrolysis probes for $\mathrm{M}$ and $\mathrm{U}$ reactions were identical, except at $\mathrm{CpG}$ sites, which were unique for recognizing $\mathrm{M}$ or $\mathrm{U}$ positions.

The amplification mixture consisted of $9.5 \mu \mathrm{l}$ of DNA Master Mix (QuantiTect Multiplex PCR Master Mix, QIAGEN), $8.4 \mu \mathrm{l}$ of $\mathrm{H} 2 \mathrm{O}, 0.125 \mu \mathrm{l}$ each primer $(280 \mathrm{ng} / \mu \mathrm{L}), 0.06 \mu \mathrm{l}$ each probe $(100 \mu \mathrm{M})$, and $3.5 \mu \mathrm{l}$ of template DNA in a final volume of $20 \mu \mathrm{l}$. PBMC methylated in vitro are used as the methylated control. qPCR reaction involved an initial denaturation at $95^{\circ} \mathrm{C}$ for $15 \mathrm{~min}$, followed by 40 cycles of $94^{\circ} \mathrm{C}$ for one min and $60^{\circ} \mathrm{C}$ for one min. Data acquisition and analysis was performed on the RQ Manager 1.2.1 software. The percentage of methylation was carried out using the following previously described [27]:

$$
\% \text { Metylation }=100 /\left(1+\left(2^{\wedge}(\text { Cq_methylated-Cq_unmethylated })\right)\right)
$$

\subsection{Statistical analysis}

The association between qualitative variables were analyzed using the chi-square test or Fisher's exact test. For the comparison between qualitative and quantitative data, the $t$-student test for independent data was used. Receiver Operating Characteristics (ROC) analysis was performed to determine the optimal cutoff for dp_qMSP. Samples were categorized as methylated or unmethylated based on the cutoff determined through ROC curve analysis. The sensitivity and specificity were obtained using MSP as the reference. In addition, time-dependent ROC curves or ROC $(t)$ were used for evaluating and comparing the prognostic value of the MGMT methylation marker between MSP and dp_qMSP[28].

The survival analysis were carried out using the Kaplan-Meier analysis and the Cox regression. In order to compare the survival functions by groups, log-rank tests were performed. Differences were considered statistically significant at $\mathrm{p} \leq 0.05$. Confidence intervals (CIs) were made using a 95\% confidence level. Statistical analysis was conducted by a biostatistical expert using the SAS 9.3 program (SAS Institute, Cary, NC, USA) and R version 4.0.0.

\section{Conclusions}

Our study presents the evaluation of two techniques used for MGMT methylation: a standard MSP and a dp_qMSP, developed in our laboratory. The dp_qMSP method used in this study was not only more sensitive but also more time-efficient for the detection of MGMT biomarker. The method is based on qPCR assay that is carried out using two fluorochrome-labeled probes to quantify the percentage of methylated molecules in the tumor sample. We concluded that this assay is highly sensitive and easy to use with a well-established cutoff point, making it reliable and suitable clinical use. Nevertheless, more multicenter studies are needed to confirm these results.

\section{Patents}

All the authors have read the journal's authorship statement and have no conflicts of interest to declare. The information provided in this study is included in a patent application process (EP19382299.6) and therefore it must be treated, solely and exclusively, based on the purposes of this paper, and should not be published if it does not respond to the purpose thereof. This application and its contents are protected by the Spanish Law on Intellectual and Industrial Property, prohibiting the distribution, reproduction, disclosure, transformation and sale of the entire document or part thereof, as well as the use, under any circumstances, of the trademarks appearing therein, without the prior express written consent of the Foundation for Biomedical Research of La Paz University Hospital-IdiPAZ (FIBHULP), which holds the ownership. 
Supplementary Materials: The following are available online at www.mdpi.com/xxx/s1, Figure S1: title, Table S1: title, Video S1: title.

Author Contributions: Conceptualization, I.IC. and R.RA.; acquisition of data for the work (data curation, methodology, validation), R.RA., J.CF., O.P., I.E., I.G., D.SC., J.C., V.M.; formal analysis and investigation, R.RA., C.RA., I.LG., IIC; resources and funding acquisition, I.IC. and J.C.; writing-original draft preparation, review and editing, R.RA., I.E., I.IC.; supervision, I.IC. All authors have read and agreed to the published version of the manuscript.

Funding: This study was supported by the "Fondo de Investigación Sanitaria-Instituto de Salud Carlos III" PI18/00050, DTS20/00029 and the European Regional Development Fund/European Social Fund FIS FEDER/FSE, Una Manera de Hacer Europa.

Acknowledgments: The authors thank Hayley Pickett from ServingMed.com for the English language correction and to the Innovation Unit from IdiPAZ for the R\&D\&I management in the patent application process associated with the technology described in this manuscript. The authors also want to recognize Repsol for providing the equipment for real time determinations.

Conflicts of Interest: The authors declare no conflict of interest.

\section{References}

1. Feinberg, A.P.; Vogelstein, B. Hypomethylation distinguishes genes of some human cancers from their normal counterparts. Nature 1983, 301, 89-92, doi:10.1038/301089a0.

2. Merlo, A.; Herman, J.G.; Mao, L.; Lee, D.J.; Gabrielson, E.; Burger, P.C.; Baylin, S.B.; Sidransky, D. 5' CpG island methylation is associated with transcriptional silencing of the tumour suppressor p16/CDKN2/MTS1 in human cancers. Nat Med 1995, 1, 686-692, doi:10.1038/nm0795-686.

3. Herman, J.G.; Baylin, S.B. Gene silencing in cancer in association with promoter hypermethylation. N Engl J Med 2003, 349, 2042-2054, doi:10.1056/NEJMra023075.

4. Esteller, M.; Silva, J.M.; Dominguez, G.; Bonilla, F.; Matias-Guiu, X.; Lerma, E.; Bussaglia, E.; Prat, J.; Harkes, I.C.; Repasky, E.A., et al. Promoter hypermethylation and BRCA1 inactivation in sporadic breast and ovarian tumors. J Natl Cancer Inst 2000, 92, 564-569, doi:10.1093/jnci/92.7.564.

5. Baylin, S.B. DNA methylation and gene silencing in cancer. Nat Clin Pract Oncol 2005, 2 Suppl 1, S4-11, doi:10.1038/ncponc0354.

6. Esteller, M.; Garcia-Foncillas, J.; Andion, E.; Goodman, S.N.; Hidalgo, O.F.; Vanaclocha, V.; Baylin, S.B.; Herman, J.G. Inactivation of the DNA-repair gene MGMT and the clinical response of gliomas to alkylating agents. N Engl J Med 2000, 343, 1350-1354, doi:10.1056/NEJM200011093431901.

7. Stupp, R.; Mason, W.P.; van den Bent, M.J.; Weller, M.; Fisher, B.; Taphoorn, M.J.; Belanger, K.; Brandes, A.A.; Marosi, C.; Bogdahn, U., et al. Radiotherapy plus concomitant and adjuvant temozolomide for glioblastoma. N Engl J Med 2005, 352, 987-996, doi:10.1056/NEJMoa043330.

8. Hegi, M.E.; Diserens, A.C.; Gorlia, T.; Hamou, M.F.; de Tribolet, N.; Weller, M.; Kros, J.M.; Hainfellner, J.A.; Mason, W.; Mariani, L., et al. MGMT gene silencing and benefit from temozolomide in glioblastoma. N Engl J Med 2005, 352, 997-1003, doi:10.1056/NEJMoa043331.

9. Jansen, M.; Yip, S.; Louis, D.N. Molecular pathology in adult gliomas: diagnostic, prognostic, and predictive markers. Lancet Neurol 2010, 9, 717-726, doi:10.1016/S1474-4422(10)70105-8.

10. Malley, D.S.; Hamoudi, R.A.; Kocialkowski, S.; Pearson, D.M.; Collins, V.P.; Ichimura, K. A distinct region of the MGMT CPG island critical for transcriptional regulation is preferentially methylated in glioblastoma cells and xenografts. Acta Neuropathol 2011, 121, 651-661, doi:10.1007/s00401-011-0803-5.

11. Bady, P.; Sciuscio, D.; Diserens, A.C.; Bloch, J.; van den Bent, M.J.; Marosi, C.; Dietrich, P.Y.; Weller, M.; Mariani, L.; Heppner, F.L., et al. MGMT methylation analysis of glioblastoma on the Infinium methylation BeadChip identifies two distinct $\mathrm{CpG}$ regions associated with gene silencing and outcome, yielding a prediction model for comparisons across datasets, tumor grades, and CIMP-status. Acta Neuropathol 2012, 124, 547-560, doi:10.1007/s00401-012-1016-2.

12. Frommer, M.; McDonald, L.E.; Millar, D.S.; Collis, C.M.; Watt, F.; Grigg, G.W.; Molloy, P.L.; Paul, C.L. A genomic sequencing protocol that yields a positive display of 5-methylcytosine residues in individual DNA strands. Proc Natl Acad Sci U S A 1992, 89, 1827-1831, doi:10.1073/pnas.89.5.1827. 
13. Christians, A.; Hartmann, C.; Benner, A.; Meyer, J.; von Deimling, A.; Weller, M.; Wick, W.; Weiler, M. Prognostic value of three different methods of MGMT promoter methylation analysis in a prospective trial on newly diagnosed glioblastoma. PLoS One 2012, 7, e33449, doi:10.1371/journal.pone.0033449.

14. Quillien, V.; Lavenu, A.; Karayan-Tapon, L.; Carpentier, C.; Labussiere, M.; Lesimple, T.; Chinot, O.; Wager, M.; Honnorat, J.; Saikali, S., et al. Comparative assessment of 5 methods (methylation-specific polymerase chain reaction, MethyLight, pyrosequencing, methylation-sensitive high-resolution melting, and immunohistochemistry) to analyze O6-methylguanine-DNA-methyltranferase in a series of 100 glioblastoma patients. Cancer 2012, 118, 4201-4211, doi:10.1002/cncr.27392.

15. Bienkowski, M.; Berghoff, A.S.; Marosi, C.; Wohrer, A.; Heinzl, H.; Hainfellner, J.A.; Preusser, M. Clinical Neuropathology practice guide 5-2015: MGMT methylation pyrosequencing in glioblastoma: unresolved issues and open questions. Clin Neuropathol 2015, 34, 250-257, doi:10.5414/np300904.

16. Yoshioka, M.; Matsutani, T.; Hara, A.; Hirono, S.; Hiwasa, T.; Takiguchi, M.; Iwadate, Y. Real-time methylation-specific PCR for the evaluation of methylation status of MGMT gene in glioblastoma. Oncotarget 2018, 9, 27728-27735, doi:10.18632/oncotarget.25543.

17. Barault, L.; Amatu, A.; Bleeker, F.E.; Moutinho, C.; Falcomata, C.; Fiano, V.; Cassingena, A.; Siravegna, G.; Milione, M.; Cassoni, P., et al. Digital PCR quantification of MGMT methylation refines prediction of clinical benefit from alkylating agents in glioblastoma and metastatic colorectal cancer. Ann Oncol 2015, 26, 1994-1999, doi:10.1093/annonc/mdv272.

18. Park, C.K.; Kim, J.; Yim, S.Y.; Lee, A.R.; Han, J.H.; Kim, C.Y.; Park, S.H.; Kim, T.M.; Lee, S.H.; Choi, S.H., et al. Usefulness of MS-MLPA for detection of MGMT promoter methylation in the evaluation of pseudoprogression in glioblastoma patients. Neuro Oncol 2011, 13, 195-202, doi:10.1093/neuonc/noq162.

19. Yamashita, S.; Yokogami, K.; Matsumoto, F.; Saito, K.; Mizuguchi, A.; Ohta, H.; Takeshima, H. MGMT promoter methylation in patients with glioblastoma: is methylation-sensitive high-resolution melting superior to methylation-sensitive polymerase chain reaction assay? J Neurosurg 2018, 130, 780-788, doi:10.3171/2017.11.JNS171710.

20. Mikeska, T.; Bock, C.; El-Maarri, O.; Hubner, A.; Ehrentraut, D.; Schramm, J.; Felsberg, J.; Kahl, P.; Buttner, R.; Pietsch, T., et al. Optimization of quantitative MGMT promoter methylation analysis using pyrosequencing and combined bisulfite restriction analysis. J Mol Diagn 2007, 9, 368-381, doi:10.2353/jmoldx.2007.060167.

21. Wenger, A.; Ferreyra Vega, S.; Kling, T.; Bontell, T.O.; Jakola, A.S.; Caren, H. Intratumor DNA methylation heterogeneity in glioblastoma: implications for DNA methylation-based classification. Neuro Oncol 2019, 21, 616-627, doi:10.1093/neuonc/noz011.

22. Weller, M.; Stupp, R.; Reifenberger, G.; Brandes, A.A.; van den Bent, M.J.; Wick, W.; Hegi, M.E. MGMT promoter methylation in malignant gliomas: ready for personalized medicine? Nat Rev Neurol 2010, 6, 39-51, doi:10.1038/nrneurol.2009.197.

23. Parker, N.R.; Hudson, A.L.; Khong, P.; Parkinson, J.F.; Dwight, T.; Ikin, R.J.; Zhu, Y.; Cheng, Z.J.; Vafaee, F.; Chen, J., et al. Intratumoral heterogeneity identified at the epigenetic, genetic and transcriptional level in glioblastoma. Sci Rep 2016, 6, 22477, doi:10.1038/srep22477.

24. Ibanez de Caceres, I.; Dulaimi, E.; Hoffman, A.M.; Al-Saleem, T.; Uzzo, R.G.; Cairns, P. Identification of novel target genes by an epigenetic reactivation screen of renal cancer. Cancer Res 2006, 66, 5021-5028, doi:10.1158/0008-5472.CAN-05-3365.

25. Ibanez de Caceres, I.; Cortes-Sempere, M.; Moratilla, C.; Machado-Pinilla, R.; Rodriguez-Fanjul, V.; Manguan-Garcia, C.; Cejas, P.; Lopez-Rios, F.; Paz-Ares, L.; de CastroCarpeno, J., et al. IGFBP-3 hypermethylation-derived deficiency mediates cisplatin resistance in non-small-cell lung cancer. Oncogene 2010, 29, 1681-1690, doi:10.1038/onc.2009.454.

26. Esteller, M.; Hamilton, S.R.; Burger, P.C.; Baylin, S.B.; Herman, J.G. Inactivation of the DNA repair gene O6-methylguanine-DNA methyltransferase by promoter hypermethylation is a common event in primary human neoplasia. Cancer Res 1999, 59, 793-797.

27. Eads, C.A.; Danenberg, K.D.; Kawakami, K.; Saltz, L.B.; Blake, C.; Shibata, D.; Danenberg, P.V.; Laird, P.W. MethyLight: a high-throughput assay to measure DNA methylation. Nucleic Acids Res 2000, 28, E32, doi:10.1093/nar/28.8.e32.

28. Heagerty, P.J.; Lumley, T.; Pepe, M.S. Time-dependent ROC curves for censored survival data and a diagnostic marker. Biometrics 2000, 56, 337-344, doi:10.1111/j.0006-341x.2000.00337.x.

29. In BEST (Biomarkers, EndpointS, and other Tools) Resource, Silver Spring (MD), 2016. 
30. Smith, C.J.; Osborn, A.M. Advantages and limitations of quantitative PCR (Q-PCR)-based approaches in microbial ecology. FEMS Microbiol Ecol 2009, 67, 6-20, doi:10.1111/j.1574-6941.2008.00629.x.

31. Thammaiah, S.; Venkobarao, M.C.; Sathyavanthan, H.; Mirnalini, A.S. Quantitative polymerase chain reaction-based detection of HPV 16 E6 and E7 DNA in oral squamous cell carcinoma. J Oral Pathol Med 2018, 47, 873-879, doi:10.1111/jop.12770.

32. Hegi, M.E.; Diserens, A.C.; Godard, S.; Dietrich, P.Y.; Regli, L.; Ostermann, S.; Otten, P.; Van Melle, G.; de Tribolet, N.; Stupp, R. Clinical trial substantiates the predictive value of O-6-methylguanine-DNA methyltransferase promoter methylation in glioblastoma patients treated with temozolomide. Clin Cancer Res 2004, 10, 1871-1874, doi:10.1158/1078-0432.ccr-03-0384.

33. Herrlinger, U.; Rieger, J.; Koch, D.; Loeser, S.; Blaschke, B.; Kortmann, R.D.; Steinbach, J.P.; Hundsberger, T.; Wick, W.; Meyermann, R., et al. Phase II trial of lomustine plus temozolomide chemotherapy in addition to radiotherapy in newly diagnosed glioblastoma: UKT-03. J Clin Oncol 2006, 24, 4412-4417, doi:10.1200/JCO.2006.06.9104.

34. Malmstrom, A.; Gronberg, B.H.; Marosi, C.; Stupp, R.; Frappaz, D.; Schultz, H.; Abacioglu, U.; Tavelin, B.; Lhermitte, B.; Hegi, M.E., et al. Temozolomide versus standard 6-week radiotherapy versus hypofractionated radiotherapy in patients older than 60 years with glioblastoma: the Nordic randomised, phase 3 trial. Lancet Oncol 2012, 13, 916-926, doi:10.1016/S1470-2045(12)70265-6.

35. Wick, W.; Platten, M.; Meisner, C.; Felsberg, J.; Tabatabai, G.; Simon, M.; Nikkhah, G.; Papsdorf, K.; Steinbach, J.P.; Sabel, M., et al. Temozolomide chemotherapy alone versus radiotherapy alone for malignant astrocytoma in the elderly: the NOA-08 randomised, phase 3 trial. Lancet Oncol 2012, 13, 707-715, doi:10.1016/S1470-2045(12)70164-X.

36. Johannessen, L.E.; Brandal, P.; Myklebust, T.A.; Heim, S.; Micci, F.; Panagopoulos, I. MGMT Gene Promoter Methylation Status - Assessment of Two Pyrosequencing Kits and Three Methylation-specific PCR Methods for their Predictive Capacity in Glioblastomas. Cancer Genomics Proteomics 2018, 15, 437-446, doi:10.21873/cgp.20102.

37. Preusser, M.; Berghoff, A.S.; Manzl, C.; Filipits, M.; Weinhausel, A.; Pulverer, W.; Dieckmann, K.; Widhalm, G.; Wohrer, A.; Knosp, E., et al. Clinical Neuropathology practice news 1-2014: pyrosequencing meets clinical and analytical performance criteria for routine testing of MGMT promoter methylation status in glioblastoma. Clin Neuropathol 2014, 33, 6-14, doi:10.5414/np300730.

38. Brigliadori, G.; Foca, F.; Dall'Agata, M.; Rengucci, C.; Melegari, E.; Cerasoli, S.; Amadori, D.; Calistri, D.; Faedi, M. Defining the cutoff value of MGMT gene promoter methylation and its predictive capacity in glioblastoma. J Neurooncol 2016, 128, 333-339, doi:10.1007/s11060-016-2116-y.

39. Malmstrom, A.; Lysiak, M.; Kristensen, B.W.; Hovey, E.; Henriksson, R.; Soderkvist, P. Do we really know who has an MGMT methylated glioma? Results of an international survey regarding use of MGMT analyses for glioma. Neurooncol Pract 2020, 7, 68-76, doi:10.1093/nop/npz039.

40. Wick, W.; Weller, M.; van den Bent, M.; Sanson, M.; Weiler, M.; von Deimling, A.; Plass, C.; Hegi, M.; Platten, M.; Reifenberger, G. MGMT testing--the challenges for biomarker-based glioma treatment. Nat Rev Neurol 2014, 10, 372-385, doi:10.1038/nrneurol.2014.100. 